\title{
Association between GDF5 rs143383 polymorphism and knee osteoarthritis: an updated meta-analysis based on 23,995 subjects
}

\author{
Feng Pan ${ }^{*}$, Jing Tian, Tania Winzenberg, Changhai Ding and Graeme Jones
}

\begin{abstract}
Background: Previous studies investigating the association between GDF5 rs143383 polymorphism and knee osteoarthritis (OA) have suggested stronger associations in Asians than Caucasians, but limitations on the amount of available data have meant that a definitive assessment has not been possible. Given the availability of more recent data, the aim of this meta-analysis was to determine the overall association between GDF5 rs143383 polymorphism and knee $O A$ and whether the association varies by ethnicity.
\end{abstract}

Methods: Searches of Medline, Embase, and ISI Web of Science were conducted up to July 2013. Summary odds ratios (ORs) and 95\% confidence intervals (Cls) were calculated to estimate the strength of association between the GDF5 polymorphism and knee OA risk.

Results: A total of 20 studies with 23,995 individuals were included. There were weak but significant associations present between the GDF5 polymorphism and knee OA at the allele level ( $\mathrm{C}$ vs. T: $\mathrm{OR}=0.85,95 \% \mathrm{Cl}=0.80-0.90)$ and genotype level (CC vs. TT: $O R=0.73 ; C T$ vs. T: $O R=0.84 ; C C / C T$ vs. T: $O R=0.81 ; C C$ vs. $C T / T$ : $O R=0.81$ ) in the overall population. In the subgroup analysis by ethnicity, we observed a strong significant association $(O R=0.60$ to 0.80 , all $P<0.05$ ) in Asian population and weaker associations ( $\mathrm{OR}=0.78$ to 0.87, all $P<0.05$ ) in Caucasian population; however marked heterogeneity was detected in all models except for CC vs. $\Pi\left(P^{2}=12.9 \%\right)$ and CC vs. $C T+\Pi\left(P^{2}=0.0 \%\right)$ in Asians.

Conclusions: These results strongly suggest that the $C$ allele and CC genotype of the GDF5 gene are protective for knee OA susceptibility across different populations.

Keywords: GDF5, Polymorphism, Knee, Osteoarthritis, Meta-analysis

\section{Background}

Osteoarthritis (OA) is the most prevalent form of arthritis in the worldwide and is regarded as a disorder of the whole joint [1,2]. One of the most frequently affected joints is the knee, with a prevalence of $30 \%$ in those over 65 years old [3]. There is a strong genetic component of OA with heritability estimates showing that genetic components account for $39-65 \%$ of the risk for the development of knee OA $[2,4,5]$. However, the identification of specific genes has been problematic with some genes associated with pain [6,7] and others with joint structures [8]. Overall, there is a lack of consistency of associations.
One of the most comprehensively studied candidate genes is growth differentiation factor 5 (GDF5). GDF5, also known as cartilage-derived morphogenetic protein 1 , is a member of the transforming growth factor- $\beta$ superfamily and closely correlated with bone morphogenetic proteins. GDF5 has been shown to be involved in musculoskeletal processes including the development, maintenance and repair of bone, cartilage and other tissues of synovial joint as well as tendon $[9,10]$. In light of the important functions of GDF5, any changes affecting a reduction in the expression of this protein could increase the risk of OA.

\footnotetext{
* Correspondence: Feng.Pan@utas.edu.au

Menzies Research Institute Tasmania, University of Tasmania, Private Bag 23, Hobart, Tasmania 7000, Australia
} 
GDF5 mutations in humans have been implicated in several disorders of skeletal development [11]. Singlenucleotide polymorphisms (SNPs) have been identified in the $5^{\prime}$ - -untranslated region ( $5^{\prime}$-UTR) of GDF5 which is involved in the regulation of GDF5 transcriptional activity [12]. As for one of the most common polymorphisms (rs143383), $\mathrm{T}$ to $\mathrm{C}$ substitution in the promoter region of GDF5 has an effect on the expression of GDF5 production, with lower GDF5 expression of the OA-associated T allele [13]. Several studies have suggested that GDF5 rs143383 polymorphism may be related to an elevated risk of OA in certain ethnic groups [12,14-16]. However, these positive associations have not been consistently replicated. For instance, two studies from Korea and Greece failed to detect any association with knee OA $[17,18]$. Two earlier meta-analyses suggested that an increase in the risk of knee OA was associated with GDF5 rs143383 polymorphism in Asians and Caucasians [11,19]. Since then, multiple studies on the relationship of knee OA with GDF5 have been published. Therefore, the aim of this study was to determine the overall association between GDF5 rs143383 polymorphism and knee OA risk and whether the association varies by ethnicity.

\section{Methods}

\section{Literature search strategy}

We searched Medline, Embase and ISI Web of science databases for all English articles on the association between GDF5 gene promoter polymorphism and OA (last report up to 13 July 2013). Combinations of keywords used in the search were: ("Growth differentiation factor 5" or "GDF5" or "rs143383" or "+104 T/C"), ("polymorphism" or "polymorphisms") and ("osteoarthritis" or "OA"). References of retrieved studies and review articles were also screened for other additional eligible publications and unpublished studies. Conference abstracts were not considered.

\section{Inclusion and exclusion criteria}

All studies included in this meta-analysis had to meet the following criteria: (1) the type of study was a casecontrol or cohort study; (2) a study investigated the association of GDF5 (rs143383; +104 T/C) polymorphism with knee $\mathrm{OA}$; (3) available alleles or genotypes frequencies of GDF5 were provided to evaluate the odds ratios (ORs) with 95\% confidence intervals (CIs). The exclusion criteria were as follows: (1) the study was not conducted on knee OA; (2) the study was conducted on animals or cells; (3) the data could not be extracted after contacting with the authors.

\section{Data extraction}

All data were extracted independently from eligible studies by two reviewers (Pan and Tian) according to the criteria listed above. The following information were collected: the first author's name, publication date, country of origin, study design, ethnicity, total sample size of cases and controls, genotype and allele frequencies of cases and controls, sources of controls, age, sex and genotyping method, which also were reviewed by a third investigator (Jones). We also extracted data on how knee OA was defined i.e. clinical criteria, radiographic criteria, or total knee replacement (TKR). For clinical criteria, the American College of Rheumatology (ACR) criteria was used if information on ACR was available [20]. For radiographic criteria, Kellgren/Lawrence (K/L) score (0-4 scale) was used to identify and grade knee OA. A cut-off of K/L score 2 was used to be a classification of knee OA [21]. Any controversies about interpretation of data were discussed within our research team to reach a consensus. In cases where the same patient population was included in different studies, only the larger sample size was included in this meta-analysis. If one study contains the results from different populations, each group was treated independently. Authors were contacted where unpublished data or clarification were needed.

\section{Statistical methods}

Allele frequencies at GDF5 rs143383 polymorphism from the respective study were determined by the allele counting method. The strength of the association between GDF5 rs143383 polymorphism and knee OA susceptibility was estimated by calculating the pooled ORs with their 95\% CIs. The Z-test was used to determine the significance of the pooled ORs and 95\% CIs. The pooled ORs were performed for additive $(\mathrm{C}$ vs. $\mathrm{T})$, co-dominant $(\mathrm{CC}$ vs. TT; $\mathrm{CT}$ vs. TT), dominant $(\mathrm{CC}+\mathrm{CT}$ vs. TT), and recessive (CC vs. CT + TT) models. The between-study heterogeneity was assessed using the Chi square based Qstatistic [22]. If a $P$ value less than 0.10 for the $Q$-test was observed, it indicates the presence of heterogeneity among studies [23]. The $I^{2}$ statistic $\left(I^{2}=100 \% \times(Q-\mathrm{df}) / Q\right)$ was also used to quantify heterogeneity. $I^{2}$ ranges from 0 to $100 \%$ which is interpreted as the degree of inconsistency across studies [24]. An $I^{2}$ greater than 50\% was considered as heterogeneity among studies. The random-effect model was used to determine the pooled ORs. Sensitivity analysis was performed by excluding the Hardy-Weinberg equilibrium (HWE)-violating studies [25]. Potential publication bias was assessed by the funnel plot, in which the standard error of $\log (\mathrm{OR})$ of each study was plotted against its log (OR). A symmetric plot indicates a low risk of publication bias. If visual inspection suggested there was funnel plot asymmetry, the method of Egger's linear regression test was used to further assess [26]. All statistical analyses were carried out using STATA version 7.0 (Stata Corporation, College Station, TX). Two-sided $P<0.05$ was considered to be statistically significant. 


\section{Results}

\section{Characteristics of included studies}

A total of 12 articles were identified [11,12,14-19,27-30]. Among these, one article [12] reported on a Japanese population and a Chinese population, these were considered as two separate studies. Two other studies performed by Southam et al. [14] and Valdes et al. [27] also included two independent studies, the former contained UK and Spanish studies, and the latter investigated two different populations in the UK. In addition, the three previous meta-analyses included unpublished data from independent studies $[11,19,30]$ where only $\mathrm{T}$ allele and $\mathrm{C}$ allele counts can be extracted from the Twins UK and Finnish study [19], and the Rotterdam study III [30]. Thus, 20 studies with 8,709 cases and 15,286 controls were included in the current metaanalysis. The study flow chart is shown in Figure 1. Detailed characteristics of these studies are listed in Tables 1 and 2. Of eligible studies, 6 studies $(n=6,219)$ and 14 studies $(\mathrm{n}=17,776)$ were conducted respectively in Asian and Caucasian populations. Male and female subgroups were available from 5 studies in Asian and 4 studies in Caucasian population.

\section{Quantitative assessment}

The summary of meta-analyses for GDF5 rs143383 polymorphism with OA is shown in Table 3.

\section{Overall population}

20 separate studies had available data for analysis of GDF5 rs143383 polymorphism and knee OA risk with a total sample size of 8,709 cases and 15,286 controls. In the allele model and genotype models, significant associations were found when all studies were pooled in the overall population (Table 3). The summary OR for allele model was 0.85 (95\% CI $=0.80-0.90)$. The forest plot of the distribution of the ORs for allele model is shown in Figure 2. Similarly, the summary ORs for genotype models ranged from 0.73 to 0.84 . There was substantial and statistically significant heterogeneity for CT vs. TT $\left(I^{2}=62.2 \%\right)$ and dominant model $\left(I^{2}=61.7 \%\right)$.

\section{Subgroup analyses by ethnicity}

Protective effects in Asian populations were consistently greater in magnitude and the associations were all statistically significant in the Asian subgroup except for $\mathrm{CT}$ versus $\mathrm{TT}$ which approached but did not reach

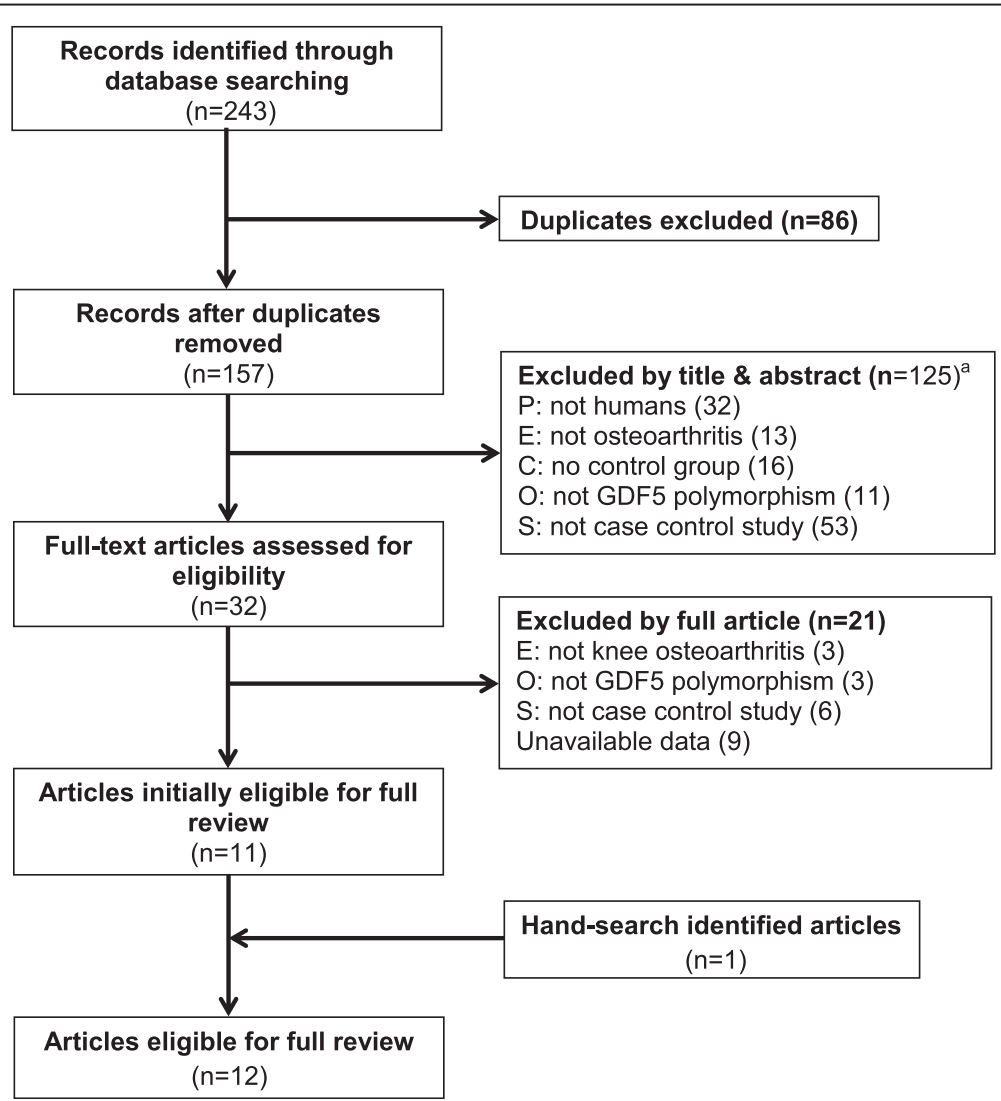

Figure 1 Flow diagram of the study selection process. ${ }^{a}$ : population group; E: exposure; C: control group; O: outcome; S: study design. 
Table 1 Characteristics of individual studies included in meta-analysis

\begin{tabular}{|c|c|c|c|c|c|c|c|c|c|c|c|c|c|}
\hline \multirow[t]{2}{*}{ First author } & \multirow[t]{2}{*}{ Year } & \multirow[t]{2}{*}{ Country } & \multirow[t]{2}{*}{ Study design } & \multirow[t]{2}{*}{ Ethnicity } & \multicolumn{2}{|c|}{ Sample size } & \multirow{2}{*}{$\begin{array}{l}\text { Source of } \\
\text { controls }\end{array}$} & \multirow[t]{2}{*}{ Genotyping method } & \multicolumn{2}{|c|}{ Age (mean) } & \multicolumn{3}{|c|}{ Knee OA definition } \\
\hline & & & & & Case & $\overline{\text { Control }}$ & & & Case & Control & Radiographic* & Clinical $^{\dagger}$ & TKR \\
\hline Southam ${ }^{a}$ & 2007 & UK & Case-control & Caucasian & 349 & 822 & $\mathrm{HB}$ & PCR-RFLP & 65 & 69 & & & + \\
\hline Southam ${ }^{b}$ & 2007 & Spain & Case-control & Caucasian & 274 & 1196 & HB & TaqMan & NA & $>55.0$ & & & + \\
\hline Miyamoto $^{a}$ & 2007 & Japan & Case-control & Asian & 718 & 861 & $H B$ & $\begin{array}{l}\text { TaqMan, Invader, DNA fragment analysis } \\
\text { or Direct sequence }\end{array}$ & 71.9 & 49.4 & + & & \\
\hline Miyamoto $^{b}$ & 2007 & China & Case-control & Asian & 313 & 485 & $H B$ & $\begin{array}{c}\text { TaqMan, Invader, DNA fragment analysis } \\
\text { or Direct sequence }\end{array}$ & 58.8 & 56.8 & + & & \\
\hline Tsezou & 2007 & Greece & Case-control & Caucasian & 251 & 268 & $H B$ & Direct sequence & 67.9 & 65.2 & + & & \\
\hline Chapman & 2008 & Netherlands & Cohort study & Caucasian & 142 & 724 & PB & Mass spectrometry & 60.4 & 59.6 & + & & \\
\hline$V$ aldes ${ }^{a}$ & 2009 & UK & Case-control & Caucasian & 735 & 654 & $H B$ & Allele-specific PCR & 68.5 & 66.9 & & + & \\
\hline Valdes ${ }^{b}$ & 2009 & UK & Cohort study & Caucasian & 264 & 512 & PB & Allele-specific PCR & 66.3 & 63 & + & & \\
\hline Vaes & 2009 & Netherlands & Cohort study & Caucasian & 667 & 2097 & PB & TaqMan & $>55.0$ & $>55.0$ & + & & \\
\hline Evangelou $^{a}$ & 2009 & Iceland & Cohort study & Caucasian & 1071 & 1169 & PB & Centaurus platform & 74.8 & 74.8 & & & + \\
\hline Evangelou ${ }^{\mathrm{b}}$ & 2009 & UK & Twins study & Caucasian & 177 & 548 & NA & Illumina platform & 54.3 & 54.3 & + & & \\
\hline Evangelou $^{c}$ & 2009 & Finland & Family-based study & Caucasian & 109 & 209 & NA & Mass spectrometry & 67 & 58 & + & & + \\
\hline Cao & 2010 & Korea & Case-control & Asian & 276 & 298 & PB & PCR-RFLP & 63 & 44 & & & + \\
\hline Tawonsawatruk & 2011 & Thailand & Case-control & Asian & 103 & 103 & $H B$ & PCR-RFLP & 68.5 & 59.25 & & & + \\
\hline Valde $^{a}$ & 2011 & UK & Cohort study & Caucasian & 867 & 758 & PB & Allele-specific PCR & 66.5 & 66.5 & & & + \\
\hline Valdes ${ }^{b}$ & 2011 & Estonian & Cohort study & Caucasian & 65 & 427 & PB & Allele-specific PCR & 47.1 & 47.1 & + & & \\
\hline Valdes $^{c}$ & 2011 & UK & Cohort study & Caucasian & 1141 & 536 & PB & Allele-specific PCR & 65.5 & 65.5 & + & & + \\
\hline Valdes $^{d}$ & 2011 & Netherlands & Cohort study & Caucasian & 162 & 1582 & PB & TaqMan & $>45.0$ & $>45.0$ & + & & \\
\hline Shin & 2012 & Korea & Cohort study & Asian & 725 & 1737 & PB & High resolution melting analysis & 67.4 & 62.7 & + & & \\
\hline Mhishra & 2013 & India & Case-control & Asian & 300 & 300 & $\mathrm{HB}$ & PCR-RFLP & 54.0 & 55.2 & + & + & \\
\hline
\end{tabular}

$\bar{a}, \mathrm{~b}, \mathrm{c}$ and ${ }^{\mathrm{d} D e n o t e}$ an independent study in the one article, respectively; NA Data not available; HB hospital-based; PB population-based; PCR-RFLP polymerase chain reaction restriction fragment length polymorphism; TKR total knee replacement.

*Radiographic criteria (Kellgren/Lawrence grade $\geq 2$ ).

${ }^{+}$Clinical criteria are based on the American College of Rheumatology. 
Table 2 Distributions of GDF5 rs 143383 genotypes and alleles among cases and controls

\begin{tabular}{|c|c|c|c|c|c|c|c|c|c|c|c|c|}
\hline \multirow[t]{2}{*}{ First author } & \multirow[t]{2}{*}{ Year } & \multicolumn{3}{|c|}{ Case } & \multicolumn{3}{|c|}{ Control } & \multicolumn{2}{|c|}{ Case } & \multicolumn{2}{|c|}{ Control } & \multirow[t]{2}{*}{$P^{H W E}$} \\
\hline & & $T T$ & $\mathrm{TC}$ & $\mathrm{CC}$ & $T \mathrm{TT}$ & TC & $\mathrm{CC}$ & $T$ & $C$ & $T$ & $C$ & \\
\hline Southam $^{a}$ & 2007 & 141 & 168 & 40 & 324 & 372 & 126 & 450 & 248 & 1020 & 624 & 0.262 \\
\hline Southam $^{b}$ & 2007 & 102 & 136 & 36 & 439 & 563 & 194 & 340 & 208 & 1441 & 951 & 0.550 \\
\hline Miyamoto ${ }^{a}$ & 2007 & 444 & 243 & 31 & 473 & 330 & 58 & 1131 & 305 & 1276 & 446 & 0.966 \\
\hline Miyamoto $^{\mathrm{b}}$ & 2007 & 197 & 97 & 19 & 244 & 193 & 48 & 491 & 135 & 681 & 289 & 0.283 \\
\hline Tsezou & 2007 & 95 & 126 & 30 & 99 & 125 & 44 & 316 & 186 & 323 & 213 & 0.669 \\
\hline Chapman & 2008 & 54 & 72 & 16 & 289 & 331 & 104 & 180 & 104 & 909 & 539 & 0.558 \\
\hline Valdes $^{\mathrm{a}}$ & 2009 & 337 & 313 & 85 & 238 & 329 & 79 & 987 & 483 & 805 & 487 & 0.032 \\
\hline Valdes $^{\mathrm{b}}$ & 2009 & 126 & 98 & 35 & 181 & 244 & 84 & 350 & 168 & 606 & 412 & 0.908 \\
\hline Vaes & 2009 & 276 & 298 & 93 & 752 & 1014 & 331 & 850 & 484 & 2518 & 1617 & 0.724 \\
\hline Evangelou $^{a}$ & 2009 & 535 & 379 & 157 & 552 & 442 & 175 & 1449 & 693 & 1546 & 792 & 0.000 \\
\hline Evangelou ${ }^{b}$ & 2009 & NA & NA & NA & NA & NA & NA & 230 & 124 & 679 & 417 & NA \\
\hline Evangelou ${ }^{c}$ & 2009 & NA & NA & NA & NA & NA & NA & 124 & 94 & 251 & 167 & NA \\
\hline Cao & 2010 & 150 & 115 & 11 & 159 & 113 & 26 & 415 & 137 & 431 & 165 & 0.360 \\
\hline Tawonsawatruk & 2011 & 38 & 41 & 11 & 33 & 47 & 23 & 117 & 63 & 113 & 93 & 0.424 \\
\hline Valdes $^{\mathrm{a}}$ & 2011 & 413 & 361 & 93 & 294 & 354 & 110 & 1187 & 547 & 942 & 574 & 0.837 \\
\hline Valdes $^{\mathrm{b}}$ & 2011 & 32 & 24 & 9 & 168 & 179 & 80 & 88 & 42 & 515 & 339 & 0.010 \\
\hline Valdes $^{c}$ & 2011 & 467 & 511 & 163 & 219 & 237 & 80 & 1445 & 837 & 675 & 397 & 0.229 \\
\hline Valdes $^{d}$ & 2011 & NA & NA & NA & NA & NA & NA & 195 & 107 & 1930 & 1234 & NA \\
\hline Shin & 2012 & 382 & 305 & 38 & 942 & 689 & 106 & 1069 & 381 & 2573 & 901 & 0.176 \\
\hline Mhishra & 2013 & 124 & 130 & 46 & 84 & 160 & 56 & 378 & 222 & 328 & 272 & 0.188 \\
\hline
\end{tabular}

significance $(P=0.071)$ (Table 3$)$. The summary ORs were highly significant especially for $\mathrm{CC}$ vs. TT $(\mathrm{OR}=0.60$, $P<0.001)$ and the recessive model $(\mathrm{OR}=0.68, P<0.001)$ (Figure 3). In the Asian subgroup, the between-study heterogeneity remained substantial apart from two models (CC vs. TT and the recessive model, $I^{2}=12.9 \%$ and $I^{2}=$ $0.0 \%$, respectively). In Caucasian populations, similar results were found under all models with weaker associations $(\mathrm{OR}=0.78$ to 0.87 , all $P<0.05)$, but a lower heterogeneity was observed.

\section{Subgroup analyses by sex}

When 9 studies with a sample size of females $(\mathrm{n}=7,203)$ and males $(n=4,733)$ were stratified by sex, there were no significant differences in effects between males and females (Table 3). In females, all models showed significant associations. A stronger significant association was observed for $\mathrm{CC}$ vs. TT $(\mathrm{OR}=0.73, P<0.001)$ in comparison with other models in males, similarly, there were significant differences for $\mathrm{CC}$ vs. TT and recessive model in females with the strongest association being for $\mathrm{CC}$ vs. TT $(\mathrm{OR}=0.65, P=0.001)$ (Figure 4$)$. Furthermore, not all associations were significant in males (CT vs. TT: OR = $0.99,95 \% \mathrm{CI}=0.81-1.20 ; \mathrm{CC} / \mathrm{CT}$ vs. $\mathrm{TT}$ : $\mathrm{OR}=0.90,95 \%$ $\mathrm{CI}=0.74-1.09)$. Intriguingly, stratification by sex reduced heterogeneity in both males and females in all models compared to that seen in the overall population. In females, substantial and statistically significant heterogeneity persisted only for CT vs. TT $\left(I^{2}=53.2 \%\right)$ and dominant models $\left(I^{2}=50.7 \%\right)$. In males, $I^{2}<50 \%$ was observed in all models.

\section{Evaluation of other potential sources of heterogeneity}

In addition to evaluation of sources of heterogeneity by ethnicity and sex, we also further investigated other potential sources of heterogeneity by control types and knee OA definition (Table 4). Subgroup analysis by control types found that heterogeneity of hospital-based group was partly attenuated with $I^{2}=0 \%$ for CC vs. TT and recessive model; however, significant heterogeneity still was seen in the population-based group. When stratification by knee OA definition, a significant reduction in the heterogeneity $\left(I^{2}<41.0 \%\right)$ was observed where TKR was used to define the cases but not for those studies using radiographic criteria.

\section{Sensitivity analyses}

Sensitivity analyses were performed by excluding the HWE-violating studies to evaluate the stability of the results. Departure from HWE was observed in the controls 
Table 3 Meta-analysis of GDF5 rs143383 polymorphism and knee OA

\begin{tabular}{|c|c|c|c|c|c|}
\hline \multirow[t]{2}{*}{ Population } & \multirow[t]{2}{*}{ Comparison $\left(\mathrm{N}^{\mathrm{a}}\right)$} & \multicolumn{2}{|c|}{ Test of association } & \multicolumn{2}{|c|}{ Test of heterogeneity } \\
\hline & & OR $(95 \% \mathrm{Cl})$ & $P^{b}$ & $P^{c}$ & $I^{2}(\%)$ \\
\hline \multirow[t]{5}{*}{ Overall } & C vs. T (20) & $0.85(0.80-0.90)$ & 0.000 & 0.019 & 44.1 \\
\hline & CC vs. TT (17) & $0.73(0.66-0.81)$ & 0.000 & 0.328 & 10.8 \\
\hline & CT vs. TT (17) & $0.84(0.76-0.94)$ & 0.002 & 0.000 & 62.2 \\
\hline & CC/CT vs. TT (17) & $0.81(0.73-0.90)$ & 0.000 & 0.000 & 61.7 \\
\hline & CC vs. CT/TT (17) & $0.81(0.74-0.86)$ & 0.000 & 0.623 & 0.0 \\
\hline \multicolumn{6}{|l|}{ Ethnicity } \\
\hline \multirow[t]{5}{*}{ Asian } & C vs. T (6) & $0.78(0.67-0.92)$ & 0.003 & 0.006 & 69.5 \\
\hline & CC vs. TT (6) & $0.60(0.48-0.76)$ & 0.000 & 0.333 & 12.9 \\
\hline & CT vs. TT (6) & $0.80(0.63-1.02)$ & 0.071 & 0.002 & 74.3 \\
\hline & CC/CT vs. TT (6) & $0.76(0.60-0.96)$ & 0.021 & 0.001 & 75.8 \\
\hline & CC vs. CT/TT (6) & $0.68(0.56-0.84)$ & 0.000 & 0.494 & 0.0 \\
\hline \multirow[t]{5}{*}{ Caucasian } & C vs. T (14) & $0.87(0.82-0.92)$ & 0.000 & 0.229 & 20.7 \\
\hline & CC vs. TT (11) & $0.78(0.70-0.87)$ & 0.000 & 0.611 & 0.0 \\
\hline & CT vs. TT (11) & $0.86(0.76-0.97)$ & 0.012 & 0.011 & 56.2 \\
\hline & CC/CT vs. TT (11) & $0.83(0.75-0.93)$ & 0.001 & 0.020 & 52.6 \\
\hline & CC vs. CT/TT (11) & $0.84(0.76-0.93)$ & 0.001 & 0.822 & 0.0 \\
\hline \multicolumn{6}{|l|}{ Sex } \\
\hline \multirow[t]{5}{*}{ Females } & C vs. T (9) & $0.85(0.78-0.93)$ & 0.000 & 0.236 & 23.3 \\
\hline & CC vs. TT (9) & $0.73(0.62-0.87)$ & 0.000 & 0.923 & 0.0 \\
\hline & CT vs. TT (9) & $0.81(0.69-0.95)$ & 0.011 & 0.029 & 53.2 \\
\hline & CC/CT vs. Tा (9) & $0.80(0.69-0.93)$ & 0.003 & 0.039 & 50.7 \\
\hline & CC vs. CT/TT (9) & $0.83(0.70-0.97)$ & 0.021 & 0.990 & 0.0 \\
\hline \multirow[t]{5}{*}{ Males } & C vs. T (9) & $0.85(0.74-0.97)$ & 0.020 & 0.171 & 30.9 \\
\hline & CC vs. TT (9) & $0.65(0.50-0.84)$ & 0.001 & 0.464 & 0.0 \\
\hline & CT vs. TT (9) & $0.99(0.81-1.20)$ & 0.888 & 0.139 & 34.8 \\
\hline & CC/CT vs. TT (9) & $0.90(0.74-1.09)$ & 0.272 & 0.107 & 39.1 \\
\hline & CC vs. CT/TT (9) & $0.66(0.51-0.84)$ & 0.001 & 0.613 & 0.0 \\
\hline
\end{tabular}

OR odds ratio, $\mathrm{Cl}$ confidence interval, ${ }^{\mathrm{a}}$ Number of comparison, ${ }^{\mathrm{b}} P$ values for within group differences were determined by $\mathrm{Z}$ test, ${ }^{\mathrm{c}} P P$ value of $Q$-test for heterogeneity test.

of three studies (Table 2). After excluding these studies, the corresponding ORs did not materially alter under all models, suggesting that the results of this meta-analysis are stable (data not shown).

\section{Evaluation of publication bias}

Begger's funnel plot was firstly performed to assess the publication bias. As shown in Figure 5, no obvious asymmetry was found by the shape of the funnel except for CC vs TT and recessive model. Egger's test was then performed for statistical test, revealing there might be publication bias under $\mathrm{CC}$ versus $\mathrm{TT}$ and recessive model.

\section{Discussion}

To our knowledge, this is the largest and most comprehensive meta-analysis to assess the association of GDF5 rs143383 polymorphism with knee OA, including data from 20 studies in 8,709 knee OA cases and 15,286 controls. Overall analysis of pooled results demonstrated a statistically significant association between the variant genotype of GDF5 and knee OA risk in all comparisons. When stratification by ethnicity, significant associations were found in Asian as well as in Caucasian populations with a greater effect sizes in Asian population, suggesting that GDF5 rs143383 polymorphism is a determinant for knee OA risk and shared between Asian and Caucasian populations.

GDF5, an extracellular signalling molecule, plays a critical role in the development, maintenance and repair of synovial joint tissues, and it has been suggested that deficiency of GDF5 is one of the most important risk factors for the pathogenesis of OA [10]. The expression of the GDF5 protein is modulated by the GDF5 gene, 


\begin{tabular}{|c|c|c|}
\hline Study & $\begin{array}{l}\text { Odds ratio } \\
(95 \% \mathrm{Cl})\end{array}$ & $\%$ Weight \\
\hline \multicolumn{3}{|l|}{ Asian } \\
\hline Miyamoto-Japan & $0.77(0.65,0.91)$ & 6.3 \\
\hline Miyamoto-China & $0.65(0.51,0.82)$ & 4.2 \\
\hline $\mathrm{CaO}$ & $0.86(0.66,1.12)$ & 3.6 \\
\hline Tawonsawatruk & $0.65(0.43,0.99)$ & 1.8 \\
\hline Shin & $1.02(0.89,1.17)$ & 7.4 \\
\hline Mhishra & $0.71(0.56,0.89)$ & 4.3 \\
\hline Subtotal & $0.78(0.67,0.92)$ & 27.7 \\
\hline \multicolumn{3}{|l|}{ Caucasian } \\
\hline Southam-UK & $0.90(0.75,1.08)$ & 5.7 \\
\hline Southam-Spain & $0.93(0.77,1.12)$ & 5.5 \\
\hline Tsezou-Greece & $0.89(0.69,1.15)$ & 3.9 \\
\hline Chapman-GARP & $0.97(0.75,1.27)$ & 3.6 \\
\hline Valdes-Nottingham & $0.81(0.69,0.95)$ & 6.7 \\
\hline Valdes-Chingford & $0.71(0.57,0.88)$ & 4.5 \\
\hline Vaes & $0.86(0.75,0.97)$ & 7.9 \\
\hline Evangelou-deCODE & $0.93(0.82,1.06)$ & 8.0 \\
\hline Evangelou-Twins UK & $0.88(0.68,1.13)$ & 3.9 \\
\hline Evangelou-Kujala & $1.14(0.82,1.59)$ & 2.6 \\
\hline Valdes-GOAL & $0.76(0.65,0.87)$ & 7.1 \\
\hline Valdes-Estonian & $0.73(0.49,1.07)$ & 1.9 \\
\hline Valdes-Hertfordshire & $0.98(0.85,1.14)$ & 6.9 \\
\hline Valdes-Rotterdam III & $0.86(0.67,1.10)$ & 4.0 \\
\hline Subtotal & $0.87(0.82,0.92)$ & 72.3 \\
\hline Overall & $0.85(0.80,0.90)$ & 100.0 \\
\hline$T$ & $T$ & \\
\hline .433666 & 2.30592 & \\
\hline \multicolumn{3}{|c|}{ Odds ratio } \\
\hline
\end{tabular}

and rare deleterious mutations in the GDF5 gene cause several disorders of skeletal development, such as chondrodysplasias and brachydactyly, suggesting this gene has a crucial role in joint homeostasis and repair [17]. Several animal models have further confirmed the evidence supporting a critical role of GDF5 [31-34]. In mice with GDF5 mutation, a number of abnormalities of joint were found including the decrease in appendicular skeleton and the limb long bones, soft tissue deformities and tendon anomaly. Taken together, these results imply that GDF5 polymorphism may have an important function in the aetiology and pathogenesis of OA.

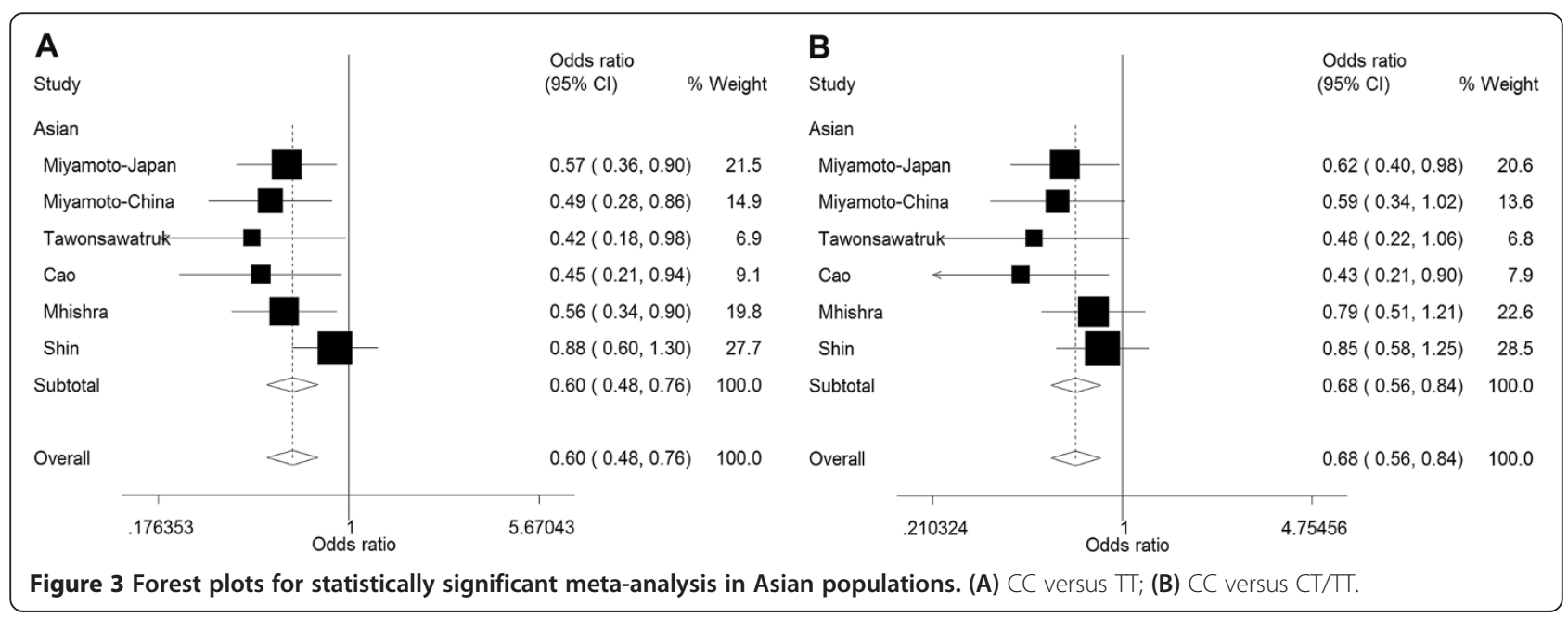




\begin{tabular}{|c|c|c|}
\hline Study & $\begin{array}{l}\text { Odds ratio } \\
(95 \% \mathrm{Cl})\end{array}$ & $\%$ Weight \\
\hline \multicolumn{3}{|l|}{ Females } \\
\hline Southam (UK) & $0.75(0.43,1.33)$ & 6.5 \\
\hline Southam (Spain) & $0.92(0.57,1.48)$ & 9.2 \\
\hline Miyamoto (Japan) & $0.61(0.35,1.06)$ & 6.9 \\
\hline Miyamoto (China) & $0.73(0.35,1.54)$ & 3.8 \\
\hline Tsezou & $0.66(0.35,1.23)$ & 5.3 \\
\hline Vaes & $0.72(0.52,0.99)$ & 20.7 \\
\hline Cao & $0.55(0.22,1.39)$ & 2.5 \\
\hline Shin & $0.94(0.57,1.56)$ & 8.2 \\
\hline Mhishra & $0.59(0.33,1.07)$ & 6.1 \\
\hline Subtotal & $0.73(0.62,0.87)$ & 69.3 \\
\hline \multicolumn{3}{|l|}{ Males } \\
\hline Southam (UK) & $0.71(0.39,1.27)$ & 6.1 \\
\hline Southam (Spain) & $0.58(0.23,1.49)$ & 2.4 \\
\hline Miyamoto (Japan) & $0.50(0.19,1.34)$ & 2.2 \\
\hline Miyamoto (China) & $0.30(0.12,0.74)$ & 2.6 \\
\hline Tsezou & $0.67(0.19,2.32)$ & 1.4 \\
\hline Vaes & $0.80(0.48,1.35)$ & 7.9 \\
\hline Cao & $0.24(0.03,1.92)$ & 0.5 \\
\hline Shin & $1.03(0.53,1.98)$ & 4.9 \\
\hline Mhishra & $0.41(0.17,0.99)$ & 2.8 \\
\hline Subtotal & $0.65(0.50,0.84)$ & 30.7 \\
\hline Overall & $0.71(0.61,0.82)$ & 100.0 \\
\hline$T$ & $T$ & \\
\hline \multirow[t]{2}{*}{.030467} & 32.8219 & \\
\hline & & \\
\hline
\end{tabular}

In this study, we found that $\mathrm{C}$ allele of GDF5 was protective for knee OA susceptibility $(\mathrm{OR}=0.85,95 \% \mathrm{CI}=$ 0.80-0.90, $P<0.001$ ), and T allele of GDF5 was associated with a higher risk for knee OA development. These findings seem to be biologically plausible. The $\mathrm{T}$ allele of the rs143383 SNP has been shown to be associated with a reduction in GDF5 transcriptional activity, thereby increasing the risk of developing knee OA, compared with the GDF5 $C$ allele $[12,14]$. In the subgroup analysis by ethnicity, effects sizes were consistently greater in Asian populations as compared to Caucasian populations, indicating that the same gene polymorphism may have different roles in knee OA susceptibility among different racial backgrounds, and the difference in linkage disequilibrium patterns may exist [19].

Several meta-analyses have been performed to identify the association between the GDF5 variant and knee OA risk. In a previous meta-analysis by Chapman et al. [11] including 2,207 cases and 4,356 controls, a significant association of GDF5 rs143383 polymorphism with knee OA was observed in Asians as well as Caucasians. In another meta-analysis, Evangelou et al. [19] included 5,085

Table 4 Identifying the source of heterogeneity by control type and knee OA definition

\begin{tabular}{|c|c|c|c|c|c|c|c|c|c|c|}
\hline \multirow[t]{2}{*}{ Subgroup } & \multicolumn{2}{|c|}{ C vs. T } & \multicolumn{2}{|c|}{ CC vs. TT } & \multicolumn{2}{|c|}{ CT vs. TT } & \multicolumn{2}{|c|}{ CC/CT vs. TT } & \multicolumn{2}{|c|}{ CC vs. $\mathrm{CT} / \mathrm{TT}$} \\
\hline & $P_{h}$ & $I^{2}(\%)$ & $P_{h}$ & $I^{2}(\%)$ & $P_{h}$ & $I^{2}(\%)$ & $P_{h}$ & $I^{2}(\%)$ & $P_{h}$ & $r^{2}(\%)$ \\
\hline \multicolumn{11}{|c|}{ Source of controls } \\
\hline $\mathrm{HB}$ & 0.196 & 29.1 & 0.681 & 0.0 & 0.013 & 60.8 & 0.032 & 54.4 & 0.703 & 0.0 \\
\hline PB & 0.051 & 46.6 & 0.233 & 23.7 & 0.006 & 62.9 & 0.005 & 64.0 & 0.511 & 0.0 \\
\hline \multicolumn{11}{|c|}{ Knee OA definition } \\
\hline Radiographic & 0.043 & 48.3 & 0.654 & 0.0 & 0.003 & 68.1 & 0.002 & 68.9 & 0.874 & 0.0 \\
\hline TKR & 0.206 & 30.5 & 0.132 & 41.0 & 0.187 & 33.3 & 0.184 & 33.6 & 0.153 & 38.0 \\
\hline
\end{tabular}

HB hospital-based, PB population-based, TKR total knee replacement, $P_{h}, P$ value of $Q$-test for heterogeneity test. 

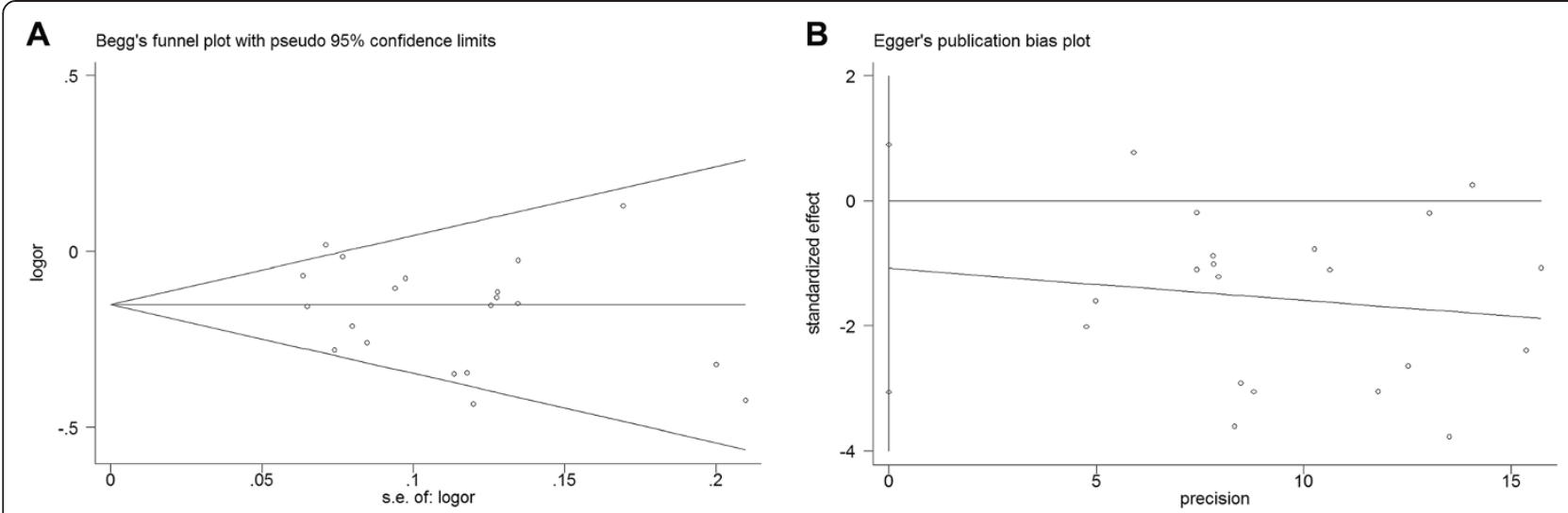

Figure 5 Funnel plot and Egger's publication bias plot for meta-analysis on association between GDF5 rs 143383 polymorphism and knee osteoarthritis risk (C versus T). (A) Begg's funnel plot for meta-analysis; (B) Egger's linear regression test for publication bias.

cases and 8,135 controls and found that GDF5 rs143383 polymorphism was associated with the risk of knee OA. In the subgroup analysis, the same association was observed across different populations. Although two recent meta-analyses also suggested that GDF5 rs143383 polymorphism was associated with knee OA susceptibility, their results should be interpreted with caution [35,36]. In the study performed by Liu et al. [35], all OA cases were pooled into their study and subgroup analyses by joint site, ethnicity, and sex were not performed. In the study by Hao and Jin [36] comprising 6 studies with 2,744 cases and 4,518 controls, there was incomplete identification of publications, which may distort the results [37]. Valdes et al. [30] also performed the meta-analysis with the largest sample size (7,579 cases and 11,947 controls), reporting that the $\mathrm{T}$ allele of the GDF5 polymorphism is associated with a $17 \%$ elevated risk for knee OA. Consistent with this, the present meta-analysis with a larger sample size showed a similar effect size of $\mathrm{T}$ allele for knee $\mathrm{OA}$ in the overall population. However, we found a slightly lower risk estimate for the $\mathrm{T}$ allele $(\mathrm{OR}=1.15)$ in Caucasian population as compared to the Valdes' paper $(\mathrm{OR}=1.16)$. This discrepancy may be due to inconsistency of reporting data from the Rotterdam I study. In the current paper, data from this study was extracted from one of the original papers reporting Rotterdam I study [28] rather than from a previous meta-analysis [19], thereby leading to a slight data variation. Additionally, the GDF5 polymorphism was found to be consistently associated with knee OA risk in Asian population. This further provides strong evidence of GDF5 rs143383 polymorphism to knee OA risk across different populations.

Heterogeneity is a potential problem in the understanding the results of meta-analyses. In this study, significant heterogeneity between different studies was observed in the overall population. To clarify the source of heterogeneity, ethnicity and sex were used to stratify the studies, finding part of this heterogeneity can be effectively attenuated or removed when stratification by sex. This indicates that it is important for meta-analyses of genetic association studies to perform subgroup analyses by sex. After subgroup analysis by source of controls, the heterogeneity was also decreased; therefore, it can be assumed that the heterogeneity partly results from difference of source of controls. That may be because potential confounding factors in many epidemiologic studies may result from the difference in control types [38]. In addition, different studies used different criteria to define the cases, which might be one of sources of heterogeneity. Some centres defined their cases using the $\mathrm{K} / \mathrm{L}$ classification and/or ACR criteria, whereas other centres used a TKR to define their cases. These differences between studies in the control group as well as key characteristics of the participants might lead to heterogeneity in the magnitude of the genetic effects [19]. Therefore, a broad consensus should be reached about OA phenotype definitions and how to enrol an ideal control group. Furthermore, other factors also should be explored to identify the source of heterogeneity if more data was available.

Of note, several potential limitations of this study should be acknowledged. Firstly, knee OA is a multifactorial disease with complex associations between genetic factors and environmental factors, and is a polygenic disease that could not be conferred significantly by no loci individually $[39,40]$. Hence, some environmental factors or other polymorphic loci should be taken into account together to arrive at a true effect of GDF5 gene. Secondly, in view of our results from unadjusted estimates, a more accurate assessment should be performed according to age, body mass index, smoking status, and other lifestyle factors if more detailed data were available. Thirdly, publication bias was found in two models, which may give rise to biased results, in particular potentially an overestimate of the effect. However, unpublished studies would need to have a 
large negative association to have sufficient weight to substantially change our results.

\section{Conclusions}

This meta-analysis suggests that GDF5 rs143383 polymorphism is highly associated with the susceptibility to knee $\mathrm{OA}$ with protective associations for the $\mathrm{C}$ allele and $\mathrm{CC}$ genotype across different populations.

\section{Competing interests}

The authors declare that they have no competing interests.

\section{Authors' contributions}

FP participated in the design of the study, literature search, data extraction, analysis and interpretation of the data and manuscript preparation. JT participated in literature search, data extraction and interpretation of the data. TW participated in interpretation of the data and manuscript preparation. CHD participated in interpretation of the data and manuscript preparation. GJ participated in the design of the study, data extraction, interpretation of the data and manuscript preparation. All authors read and approved the final manuscript.

\section{Acknowledgments}

We would like to thank all the people who helped us in this study, especially Ana M Valdes, who sent us their original data.

Received: 18 November 2013 Accepted: 6 November 2014

Published: 2 December 2014

\section{References}

1. Litwic A, Edwards MH, Dennison EM, Cooper C: Epidemiology and burden of osteoarthritis. Br Med Bull 2013, 105:185-199.

2. Hochberg MC, Yerges-Armstrong L, Yau M, Mitchell BD: Genetic epidemiology of osteoarthritis: recent developments and future directions. Curr Opin Rheumatol 2013, 25:192-197.

3. Jones G, Ding C, Scott F, Cicuttini F: Genetic mechanisms of knee osteoarthritis: a population based case-control study. Ann Rheum Dis 2004, 63:1255-1259.

4. Loughlin J: The genetic epidemiology of human primary osteoarthritis: current status. Expert Rev Mol Med 2005, 7:1-12.

5. Spector TD, Cicuttini F, Baker J, Loughlin J, Hart D: Genetic influences on osteoarthritis in women: a twin study. BMJ 1996, 312:940-943.

6. Valdes AM, De Wilde G, Doherty SA, Lories RJ, Vaughn FL, Laslett LL, Maciewicz RA, Soni A, Hart DJ, Zhang W, Muir KR, Dennison EM, Wheeler M, Leaverton P, Cooper C, Spector TD, Cicuttini FM, Chapman V, Jones G, Arden NK, Doherty M: The lle585Val TRPV1 variant is involved in risk of painful knee osteoarthritis. Ann Rheum Dis 2011, 70:1556-1561.

7. Neogi T, Soni A, Doherty SA, Laslett LL, Maciewicz RA, Hart DJ, Zhang W, Muir KR, Wheeler M, Cooper C, Spector TD, Cicuttini F, Jones G, Nevitt M, Liu Y, Arden NK, Doherty M, Valdes AM: Contribution of the COMT Val158Met variant to symptomatic knee osteoarthritis. Ann Rheum Dis 2013, 73:315-317.

8. Sandell $\sqcup$ : Etiology of osteoarthritis: genetics and synovial joint development. Nat Rev Rheumatol 2012, 8:77-89.

9. Nishitoh H, Ichijo H, Kimura M, Matsumoto T, Makishima F, Yamaguchi A, Yamashita H, Enomoto S, Miyazono K: Identification of type I and type II serine/threonine kinase receptors for growth/differentiation factor-5. J Biol Chem 1996, 271:21345-21352.

10. Francis-West PH, Abdelfattah A, Chen P, Allen C, Parish J, Ladher R, Allen S, MacPherson S, Luyten FP, Archer CW: Mechanisms of GDF-5 action during skeletal development. Development 1999, 126:1305-1315.

11. Chapman K, Takahashi A, Meulenbelt I, Watson C, Rodriguez-Lopez J, Egli R, Tsezou A, Malizos KN, Kloppenburg M, Shi DQ, Southam L, van der Breggen R, Donn R, Qin JH, Doherty M, Slagboom PE, Wallis G, Kamatani N, Jiang Q Gonzalez A, Loughlin J, lkegawa S: A meta-analysis of European and Asian cohorts reveals a global role of a functional SNP in the 5 ' UTR of GDF5 with osteoarthritis susceptibility. Hum Mol Genet 2008, 17:1497-1504.

12. Miyamoto Y, Mabuchi A, Shi DQ, Kubo T, Takatori Y, Saito S, Fujioka M, Sudo A, Uchida A, Yamamoto S, Ozaki K, Takigawa M, Tanaka T, Nakamura Y, Jiang Q, Ikegawa S: A functional polymorphism in the 5 ' UTR of GDF5 is associated with susceptibility to osteoarthritis. Nat Genet 2007, 39:529-533.
13. Egli RJ, Southam L, Wilkins JM, Lorenzen I, Pombo-Suarez M, Gonzalez A, Carr A, Chapman K, Loughlin J: Functional analysis of the osteoarthritis susceptibility-associated GDF5 regulatory polymorphism. Arthritis Rheum 2009, 60:2055-2064

14. Southam L, Rodriguez-Lopez J, Wilkins JM, Pombo-Suarez M, Snelling S, Gomez-Reino JJ, Chapman K, Gonzalez A, Loughlin J: An SNP in the 5 '-UTR of GDF5 is associated with osteoarthritis susceptibility in Europeans and with in vivo differences in allelic expression in articular cartilage. Hum Mol Genet 2007, 16:2226-2232.

15. Tawonsawatruk T, Changthong T, Pingsuthiwong S, Trachoo O, Sura T, Wajanavisit W: A genetic association study between growth differentiation factor 5 (GDF 5) polymorphism and knee osteoarthritis in Thai population. J Orthop Surg Res 2011, 6:47.

16. Mishra A, Sanghi D, Sharma AC, Raj S, Maurya SS, Avasthi S, Singh A, Parmar D, Srivastava RN: Association of polymorphism in growth and differentiation factor 5 gene with osteoarthritis knee. Am J Biochem Biotechnol 2013, 9(1):1-7.

17. Tsezou A, Satra M, Oikonomou P, Bargiotas K, Malizos KN: The growth differentiation factor 5 (GDF5) core promoter polymorphism is not associated with knee osteoarthritis in the Greek population. J Orthopaed Res 2008, 26:136-140.

18. Shin MH, Lee SJ, Kee SJ, Song SK, Kweon SS, Park DJ, Park YW, Lee SS, Kim TJ: Genetic association analysis of GDF5 and ADAM12 for knee osteoarthritis. Joint Bone Spine 2012, 79:488-491.

19. Evangelou E, Chapman K, Meulenbelt I, Karassa FB, Loughlin J, Carr A, Doherty M, Doherty S, Gomez-Reino JJ, Gonzalez A, Halldorsson BV, Hauksson VB, Hofman A, Hart DJ, Ikegawa S, Ingvarsson T, Jiang Q, Jonsdottir I, Jonsson H, Kerkhof HJM, Kloppenburg M, Lane NE, Li J, Lories RJ, van Meurs JBJ, Nakki A, Nevitt MC, Rodriguez-Lopez J, Shi DQ, Slagboom E, et al: Large-scale analysis of association between GDF5 and FRZB variants and osteoarthritis of the Hip, knee, and hand. Arthritis Rheum 2009, 60:1710-1721.

20. Altman R, Asch E, Bloch D, Bole G, Borenstein D, Brandt K, Christy W, Cooke TD, Greenwald R, Hochberg M, Howell D, Kaplan D, Koopman W, Longley S III, Mankin H, Mcshane DJ, Medsger T Jr, Meenan R, Mikkelsen W, Mqskowitz R, Murphy W, Rothschild B, Segal M, Sokoloff L, Wolfe F: Development of criteria for the classification and reporting of osteoarthritis. Classification of osteoarthritis of the knee. Diagnostic and Therapeutic Criteria Committee of the American Rheumatism Association. Arthritis Rheum 1986, 29:1039-1049.

21. Kellgren JH, Lawrence JS: Radiological assessment of osteo-arthrosis. Ann Rheum Dis 1957, 16:494-502.

22. Cochran WG: The combination of estimates from different experiments. Biometrics 1954, 10:101-129.

23. Dersimonian R, Laird N: Metaanalysis in clinical-trials. Control Clin Trials 1986, 7:177-188

24. Higgins JPT, Thompson SG: Quantifying heterogeneity in a meta-analysis. Stat Med 2002, 21:1539-1558.

25. Thakkinstian A, McElduff P, D'Este C, Duffy D, Attia J: A method for meta-analysis of molecular association studies. Stat Med 2005, 24:1291-1306.

26. Egger M, Smith GD, Schneider M, Minder C: Bias in meta-analysis detected by a simple, graphical test. Brit Med J 1997, 315:629-634.

27. Valdes AM, Spector TD, Doherty S, Wheeler M, Hart DJ, Doherty M: Association of the DVWA and GDF5 polymorphisms with osteoarthritis in UK populations. Ann Rheum Dis 2009, 68:1916-1920.

28. Vaes RB, Rivadeneira F, Kerkhof JM, Hofman A, Pols HA, Uitterlinden AG, van Meurs JB: Genetic variation in the GDF5 region is associated with osteoarthritis, height, hip axis length and fracture risk: the Rotterdam study. Ann Rheum Dis 2009, 68:1754-1760

29. Cao Z, Lee HS, Song JH, Yoon JW, Park YK, Nam SW, Lee JY, Park WS: Growth differentiation factor 5 (GDF5) core promoter polymorphism is not associated with susceptibility to osteoarthritis of the knee in the Korean population. The Korean J Pathol 2010, 44:404-409.

30. Valdes AM, Evangelou E, Kerkhof HJ, Tamm A, Doherty SA, Kisand K, Kerna I, Uitterlinden A, Hofman A, Rivadeneira F, Cooper C, Dennison EM, Zhang W, Muir KR, loannidis JP, Wheeler M, Maciewicz RA, van Meurs JB, Arden NK, Spector TD, Doherty M: The GDF5 rs 143383 polymorphism is associated with osteoarthritis of the knee with genome-wide statistical significance. Ann Rheum Dis 2011, 70:873-875.

31. Storm EE, Huynh TV, Copeland NG, Jenkins NA, Kingsley DM, Lee SJ: Limb alterations in brachypodism mice Due to mutations in a New member of the Tgf-beta-superfamily. Nature 1994, 368:639-643. 
32. Settle SH, Rountree RB, Sinha A, Thacker A, Higgins K, Kingsley DM: Multiple joint and skeletal patterning defects caused by single and double mutations in the mouse Gdf6 and Gdf5 genes. Dev Biol 2003, 254:116-130.

33. Harada M, Takahara M, Zhe P, Otsuji M, luchi Y, Takagi M, Ogino T: Developmental failure of the intra-articular ligaments in mice with absence of growth differentiation factor 5. Osteoarthr Cartilage 2007, 15:468-474

34. Mikic B, Clark RT, Battaglia TC, Gaschen V, Hunziker EB: Altered hypertrophic chondrocyte kinetics in GDF-5 deficient murine tibial growth plates. J Orthopaed Res 2004, 22:552-556.

35. Liu J, Cai W, Zhang HX, He C, Deng LF: Rs143383 in the Growth Differentiation Factor 5 (GDF5) Gene Significantly Associated with Osteoarthritis (OA)-A Comprehensive Meta-analysis. Int J Med Sci 2013, 10:312-319.

36. Hao SW, Jin QH: Association between the $+104 \mathrm{~T} / \mathrm{C}$ polymorphism in the 5 ' UTR of GDF5 and susceptibility to knee osteoarthritis: a meta-analysis. Mol Med Rep 2013, 7:485-488.

37. Tian J, Pan F, Pan YY, Zhang Y: MDM2 SNP309 polymorphism and colorectal cancer risk: appraisal of a recent meta-analysis. DNA Cell Biol 2012, 31:269-270.

38. Ruano-Ravina A, Perez-Rios M, Barros-Dios JM: Population-based versus hospital-based controls: are they comparable? Gac Sanit 2008, 22:609-613.

39. Dodd AW, Syddall CM, Loughlin J: A rare variant in the osteoarthritisassociated locus GDF5 is functional and reveals a site that can be manipulated to modulate GDF5 expression. Eur J Hum Genet 2013, 21:517-521.

40. Reynard LN, Loughlin J: Insights from human genetic studies into the pathways involved in osteoarthritis. Nat Rev Rheumatol 2013, 9:573-583.

doi:10.1186/1471-2474-15-404

Cite this article as: Pan et al:: Association between GDF5 rs143383

polymorphism and knee osteoarthritis: an updated meta-analysis based on 23,995 subjects. BMC Musculoskeletal Disorders 2014 15:404

\section{Submit your next manuscript to BioMed Central and take full advantage of:}

- Convenient online submission

- Thorough peer review

- No space constraints or color figure charges

- Immediate publication on acceptance

- Inclusion in PubMed, CAS, Scopus and Google Scholar

- Research which is freely available for redistribution 